\title{
Malignant Perivascular Epithelioid Cell Neoplasm (Pecoma) Of Paranephric Space - A Case Report
}

\author{
Bibhas Chandra Mukhopadhyay ${ }^{1}$, Saptarshi Mukhejee ${ }^{2}$, Hanumant Singh $^{3}$, \\ Tapan Kumar Mondal ${ }^{4}$, Krishnendu Maity \\ Department of Urology, Nilratan Sircar Medical College And Hospital, Kolkata, India.
}

\begin{abstract}
Perivascular epithelioid cell neoplasms (PEComa) are rare mesenchymal tumor that can occur any part of body and have unpredictable biological behavior . They are usually benign but can be malignant. We present a case of MALIGNANT EPITHELIOID ANGIOMYOLIPOMA , a type of malignant PEComa, an exceedingly rare diagnosis. We are reporting this case not only because of rarity but difficulty in diagnosis and management of this malignancy as till date there is hardly any standard guideline on its management.
\end{abstract}

Keywords: PEComa,epithelioid,angiomyolipoma,malignant.

\section{Introduction}

The World Health Organisation defined PEComas as "a mesenchymal tumour composed of histologically and Immunohistochemically distinctive perivascular epithelioid cells". In 1996 Zamboni et al first described a group of mesenchymal tumor with presence of PEC to be known as PECOma.The Perivascular Epithelioid Cells(PEC) is a cell type with distinctive morphological, immunohistochemical, ultrastructural and genetic features and they are constantly present in PEComas that includes angiomyolipoma, clear cell sugar tumor (CCST), lymphangioleiomyomatosis etc. The PEC cells have clear to granular cytoplasm , a round to oval centrally located nucleus and an inconspicuous nucleolus. Immunohistochemically PEC expresses myogenic and melanocytic markers viz SMA(smooth muscle actin), HMB-45, MELAN-A, Desmin,Vimentin,S-100, Hmsa 1 etc.

Depending on their biological behavior Folpe et al have classified PEComas as below-

\begin{tabular}{ll}
$\begin{array}{l}\text { Table } 1 \\
\text { Proposed dassification of PEComas }\end{array}$ \\
\hline & Criteria \\
\hline Benign & Non-infiltrative \\
& Non-high nuclear grade and cellularity \\
& Mitotic rate $\leq 1 / 50 \mathrm{HPF}$ \\
& No necrosis \\
& No vascular invasion \\
& One or both of the following features: \\
& Nuclear pleomorphismimultinucieated giant cells \\
& $>5$ cm in diameter \\
Uncertain & Two or more of the following features: \\
malignant potential & $5 \mathrm{~cm}$ in diameter \\
Malignant & Infiltrative \\
High nuclear grade and cellularity \\
Mitotic rate $\geq 1 / 50$ HPF \\
Necrosis \\
Vascular invasion
\end{tabular}

Case History-a 35 years male patient presented with complain of acute flank pain of one week duration, CECT scan whole abdomen revealed a large 40x49x55 mm heterogeneously enhancing mass in left paranephric space abutting upper pole of left kidney, lower pole of spleen and tail of pancreas. 

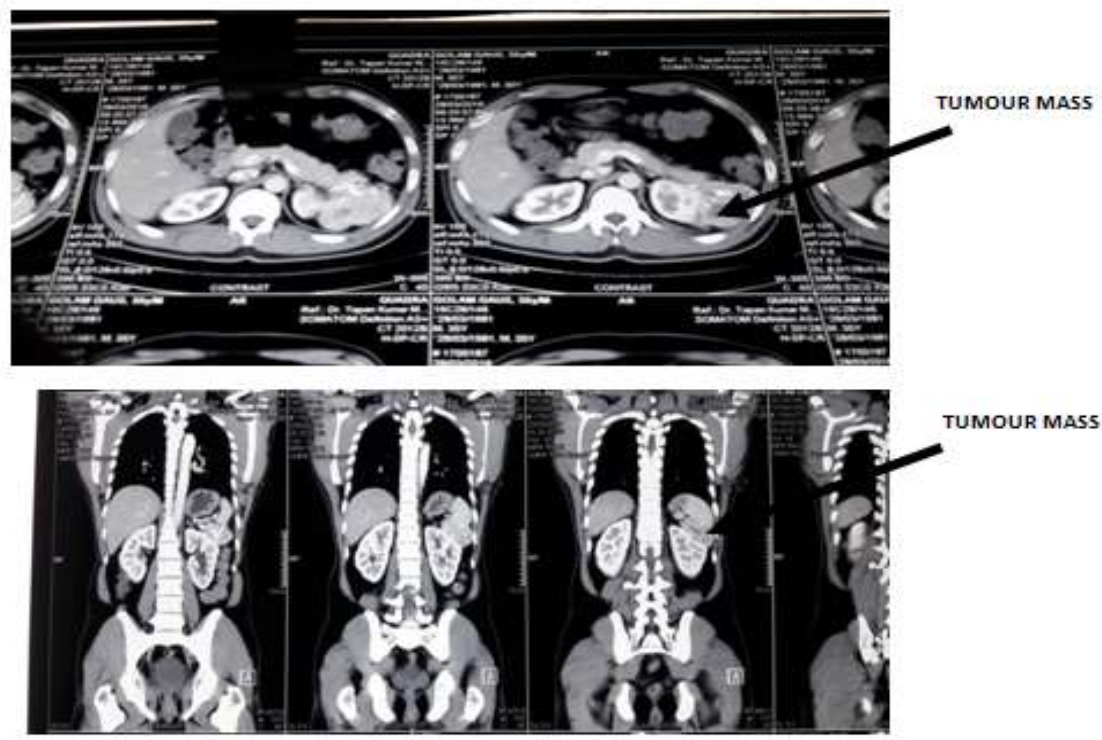

CECT scan abdomen-a exophytic heterogeneously enhancing mass in left paranephric space abutting left kidney , spleen and tail of pacreas

With CT guided trucut biopsy histopathological report showed, tumor tissue composed of malignant epithelial cells in sheets, which are mostly polygonal with abundant eosinophilic or vacuolated PAS positive cytoplasm ,mild to moderately pleomorphic vesicular nuclei distinct nucleoli. Focally spindling of tumor cells are also noted - suggestive of a malignant neoplasm . Now , on immunohistochemical examination neoplastic cells were intensely immunoreactive for Vimentin, SMA, HMB-45, ,weakly immunoreactive for Desmin but negative for pan-cytokeratin. This immunoprofile favours neoplasm of perivascular epithelioid cells (PEComa)-a epithelioid angiomyolipoma .On laparotomy through a Chevron incision there was a large retroperitoneal mass in left paranephric space distinctly separate from the tail of pancreas and spleen but there was flimsy easily separable adhesion with upper pole of left kidney with a very small, $<5 \mathrm{~mm}$,suspicious area of infiltration . A complete wide exicision of the mass with wedge resection of upper pole of left kidney including the suspicious area of infiltration was done. A slice of tissue from left renal resection bed and a part of adjacent fascia of Gerota sent separately for biopsy to assess microscopic resection margin

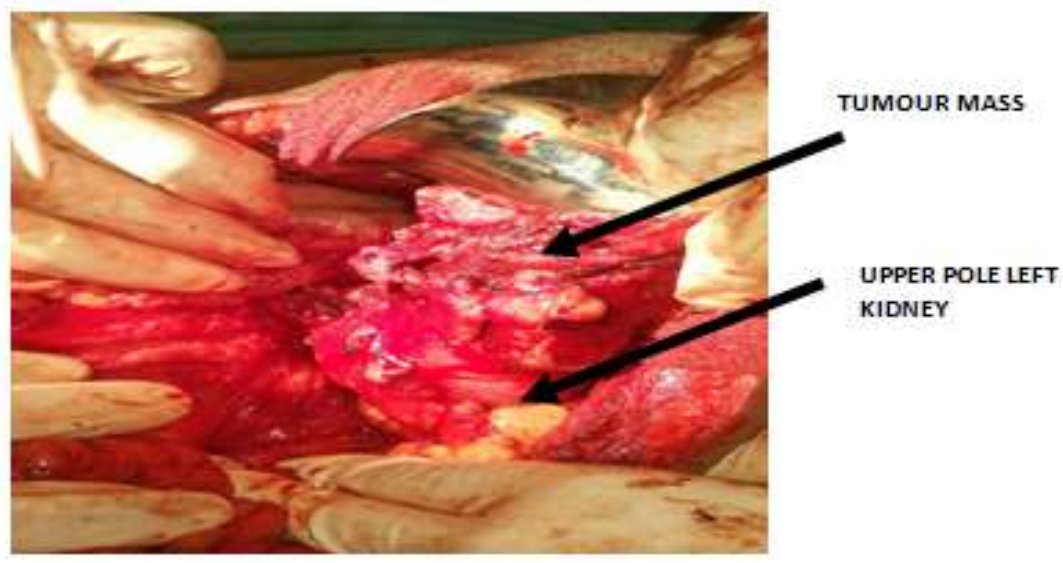

FIG 2 showing the tumor mass adhered to the upper pole of left kidney

Grossly the tumor was a partly encapsulated mass measuring $6 \times 5 \times 5 \mathrm{~cm}$ variegated with grayish white areas along with areas of hemorrhage and necrosis. On microscopic examination the tumor composed of sheets of epithelioid cells containing clear to granular eosinophilic cytoplasm, vesicular chromatin and prominent nucleoli with presence of mitosis (10-15/50HPF) areas of hemorrhage and necrosis .Focal area showed perivascular cuffing of tumor cells with multinucleated giant cells and infiltration of tumor capsule. This histopathological feature was suggestive of a malignant epithelioid angiomyolipoma a type of PEComa, there was also infiltration of left renal resection margin and fascia of Gerota. 


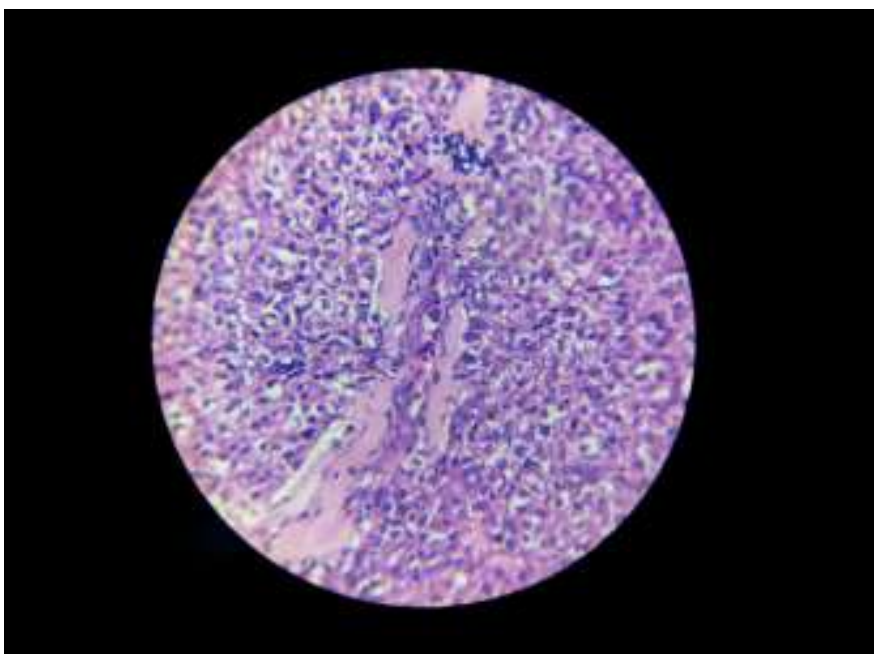

Fig 2 .Microscopic histopathological characteristics of the malignant epithelioid angiomyolipoma(malignant pecoma)

So ,the tumor came out to be a left paranephric malignant PEComa with infiltration in Gerota fascia and positive left renal resection margin and we reevaluated the case for reexploration for left radical nephrectomy as R0 resection is the only possible curative treatment of this malignancy.

\section{Discussion}

Though classical renal angiomyolipoma is the most common type of PEComa with a prevalence of $0.13 \%$ but epithelioid angiomyolipoma that is with predominant epithelioid component and poor fat content is exceedingly rare.This type of PEComa was first described by Mai et al in 1996. In 1997 Eble et al published a report of five patients with epithelioid angimyolipoma. The epithelioid cells are characteristically pancytokeratin negative and HMB-45 positive.Kato et al in 2009 described malignant epithelioid angiomyolipoma that metastasizes.According to classification of Folpe et al,as described previously,our patient's tumor had five criteria suggestive of malignancy, $>5 \mathrm{~cm}$ diameter,infiltrative growth,mitosis $>1 / 50 \mathrm{HPF}$, presence of necrosis, high nuclear grade. As chemotherapy and radiotherapy tilldate has no proven efficacy in the treatment of these tumors complete surgical (R0) resection of the tumor is the only available treatment option with curative intent.

\section{Conclusion}

After primary wide local excision of the tumor as the left renal resection margin came to be microscopically positive and fascia of Gerota was involved, reexploration with left radical nephrectomy was done. The patient is kept under follow up by clinical examination along with radiological imaging.

\section{Conflict Of Interest Statement}

All the authors declare that there are no financial or personal conflicts of interest.

\section{References}

[1]. Folpe AL, McKenney JK Li Z, Smith Smith SJ, Weiss SW. Clear cell myomelanocytic tumor of the thigh. Am J Surg Pathol. 2002;26(6):809-812

[2]. http://dx.doi.org/10.1097/00000478-200206000-00018.

[3]. Folpe AL, Mentzel T, Lehr H-A Fisher C, Balzer BL, Weiss SW. Perivascular epithelioid cell neoplasm of soft tissue and gynecologic origin. Am J Surg Pathol. 2005;29(12):1558-1575. http://dx.doi.org?10.1097/01.pas.000017323222117.37.

[4]. Hornick JL, Fletcher CDM, PEComa: what do we know so far? Hispathology. 2006;48(1) 75-82. http://dx.doi.org/10.1111/j.13652559.2005.02316.x

[5]. Wagner Aj. Malinowska- Kolodziej I, Morgam JA, et al. Clinical activity of mTOR inhibition with sirolimus in malignant periavascular epithelioid cell tumors: targeting the pathogenic activation of mTORC1 in tumors. J Clin Oncol. 2010;28(5): 835-840. http://dx.doi.org/10.1200/fCO.2009.25.2981

[6]. Pure epitheliod PECOmas (so called epithelioid angiomyolipoma) of the kidney: A clinicopathologic study of 41 cases : detailed assessment of morphology and risk stratification. Nese N, Martignoni G, Fletcher CD, Gupta R, Pan CC, Kim H etal. Am J Surg Pathol. $2011 ; 35: 161-76$.

[7]. Clinical presentation of epithelioid angiomyolipoma. Park HK, Zhang S wong MK, Kim HL. Int J Urol/ 2007; 14:21-5. 